\title{
Nicorandil attenuates endothelial VCAM-1 expression via thioredoxin production in diabetic rats induced by streptozotocin
}

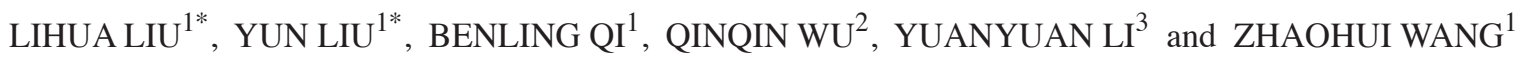 \\ ${ }^{1}$ Department of Geriatrics, Union Hospital, Tongji Medical College, \\ Huazhong University of Science and Technology, Wuhan 430022; \\ ${ }^{2}$ Department of Geriatrics, The Central Hospital of Wuhan, Wuhan 430014; \\ ${ }^{3}$ Department of Geriatrics, Wuhan General Hospital of Guangzhou Military, Wuhan 430070, P.R. China
}

Received July 8, 2013; Accepted February 27, 2014

DOI: $10.3892 / \mathrm{mmr} .2014 .2066$

\begin{abstract}
The anti-angina agent nicorandil has been reported to be beneficial even in patients who have angina with diabetes. However, the mechanism underlying the effect of nicorandil in patients with diabetes remains to be elucidated. In this study, the protective effect of nicorandil on thioredoxin (TRX) protein was investigated, as TRX is a multifunctional endogenous redox regulator that protects cells against various types of cellular and tissue stress. This study was conducted to examine whether nicorandil induces the expression of TRX for the protection against diabetic damage in the vascular tissue of rats. Diabetes was induced in rats by intraperitoneal injection of streptozotocin (STZ) (fasting glucose levels in STZ-induced rats were $>14 \mathrm{mmol} / \mathrm{l})$. Diabetic rats were divided into a diabetic control and a nicorandil-treated group. Nicorandil was administered at a dosage of $15 \mathrm{mg} / \mathrm{kg} /$ day by gavage feeding. After five weeks of nicorandil administration, blood samples were obtained from the angular vein to measure levels of stress markers, serum superoxide dismutase and malondialdehyde, using the ELISA. The expression of TRX in STZ-induced rat vascular tissue was analyzed by immunohistochemistry, quantitative polymerase chain reaction (qPCR) and western blot analysis. The oral administration of nicorandil induced TRX protein and mRNA expression in the vascular tissue of STZ-induced diabetic rats. In the diabetic control group, the levels of stress were markedly higher than those in the nicorandil-treated group, indicating that nicorandil reduces oxidative stress in serum. In addition to inducing
\end{abstract}

Correspondence to: Professor Benling Qi, Department of Geriatrics, Union Hospital, Tongji Medical College, Huazhong University Science and Technology, 1277 Jiefang Avenue, Wuhan, Hubei 430022, P.R. China

E-mail: qibenlingok@163.com

${ }^{*}$ Contributed equally

Key words: nicorandil, diabetic rats, oxidative stress, thioredoxin system, endothelial dysfunction
TRX expression, nicorandil attenuated the expression of the vascular cell adhesion molecule-1 (VCAM-1) in diabetic rat vascular endothelial cells. In conclusion, nicorandil attenuates the formation of reactive oxygen species and induces TRX protein expression, consequently resulting in the suppression of VCAM-1 secretion in the vascular endothelial cells of STZ-induced diabetic rats.

\section{Introduction}

Oxidative stress is considered a key precipitating factor in the development of diabetic complications, including diabetic cardiomyopathy (1) and diabetic vascular dysfunction (2). Not only is the generation of reactive oxygen species (ROS) elevated in diabetes, but the activity of the antioxidant defense system also declines. An increasing number of studies on diabetes and the thioredoxin (TRX) system demonstrate that levels of thioredoxin interacting protein (TXNIP), as an endogenous inhibitor of TRX, are increased in hyperglycemia. These increased TRX levels then inhibit the function of TRX, inducing increased levels of freely diffusible molecular hydrogen peroxide which contribute to oxidative stress (3-5). Therefore, TRX and TXNIP have an important role in the development of diabetes.

TRX is a $12-\mathrm{kDa}$ protein with redox-active dithiol at the active site Trp-Cys-Gly-Pro-Cys, and constitutes a major thiol reducing system. The highly catalytic motif reduces oxidized proteins and ROS, which decreases levels of oxidative stress and oxidative stress-induced damage to endothelial cells, enhances cell survival and function, and promotes endothelial cell migration and proliferation (6). Yamawaki et al (7) have shown that shear stress caused by physiological fluids exerts an atheroprotective effect in vivo by decreasing TXNIP expression and limiting pro-inflammatory events, thus improving the function of endothelial cells (7). These studies indicate that TRX and TXNIP exert a direct effect on endothelial cell function.

Nicorandil is currently undergoing clinical trials and is increasingly used for the treatment of angina. The Impact of Nicorandil in Angina (IONA) study and the Japanese Coronary Artery Disease (JCAD) study have shown that nicorandil administration in patients with coronary artery 
diseases, such as stable angina, acute myocardial infarction (MI), unstable angina and ischemic heart disease (IHD) in patients with diabetes, improves the outcome of these diseases (9-10). Being a potassium channel-opener, nicorandil activates adenosine triphosphate-sensitive potassium (K-ATP) channels (11). By expressing recombinant K-ATP channels in Xenopus oocytes, recording the macroscopic current in excised membrane patches and measuring the effects of drugs and nucleotides, it has been shown that nicorandil activates Kir6.2/sulfonylurea receptor 2A (SUR2A) and Kir6.2/SUR2B, but not Kir6.2/SUR1 currents, consistent with its specificity for cardiac and smooth muscle K-ATP channels (12); however, there is a lack of evidence in vivo. Concurrently, nicorandil improves diabetes and rat islet $\beta$-cell damage induced by streptozocin (STZ) via a radical-scavenging effect in vivo and in vitro (13). In addition, when STZ-induced diabetic rats were treated with nicorandil, its antioxidative effects prevented endothelial dysfunction via the normalization of NADPH oxidase and nitric oxide synthase (NOS) (14). However, studies investigating the interaction between nicorandil and VCAM-1, or the interaction between nicorandil and the TRX system, are not available.

On the basis of these studies, it was hypothesized that nicorandil may exert a protective effect on endothelial dysfunction through regulation of TRX and TXNIP. VCAM-1 was selected as an endothelial dysfunction biomarker.

\section{Materials and methods}

Experimental animals. Male Sprague Dawley rats (150-200 g, $\mathrm{n}=20$ ) were obtained from the Wuhan University Center for Animal Experiments/A3-Lab (Wuhan, China) and maintained in a specific pathogen-free environment with a 12-h light/dark cycle. STZ (Sigma Chemical Co., St. Louis, MO, USA) was used to induce diabetes. One group of rats $(n=14)$ received a single $50 \mathrm{mg} / \mathrm{kg}$ intraperitoneal injection of STZ in $10 \mathrm{mmol} / \mathrm{l}$ citrate buffer ( $\mathrm{pH} 4.5)(15)$. The control rats $(\mathrm{n}=6)$ were injected with citrate buffer alone. Diabetic rats were confirmed by a fasting blood glucose level (fasting $>12 \mathrm{~h}$ ) of $>14 \mathrm{mmol} / 1$ $48 \mathrm{~h}$ following STZ injection (13). One rat died 48 and $72 \mathrm{~h}$ following intraperitoneal injection, respectively.

All procedures and experiments were carried out in accordance with the principles of laboratory animal care as described by the National Institutes of Health (NIH Publication No. 85-23, revised 1996). Animal procedures, including the administration of anesthesia, were approved by the ethics committee of Huazhong University of Science and Technology (Wuhan, China).

Nicorandil administration. Rats were observed for 7 days after intraperitoneal injection of STZ, which allowed exclusion of rats that died or of those with a fasting blood glucose level that did not reach $14 \mathrm{mmol} / \mathrm{l}$. Subsequently, diabetic rats $(n=12)$ were divided into two groups. One group was used as the diabetic control and treated with a placebo $(n=6)$, and the other was treated by gavage feeding with nicorandil (Union Hospital, Wuhan, China) at a dosage of $15 \mathrm{mg} / \mathrm{kg} /$ day $(n=6)(15)$. One rat in the nicorandil-treated group $(n=5)$ died for unknown reasons after two weeks of gavage feeding. Nicorandil was administered for five weeks. Blood glucose levels were measured every seven days in each group using glucose oxidase (Nanjing Jiancheng Bioengineering Institute, Nanjing, China) with blood extracted from the tail veins. Additional blood samples were obtained from the angular vein through a capillary siphon following fasting for $>12 \mathrm{~h}$. All animals were sacrificed after five weeks of nicorandil administration. The rats were anesthetized with $2 \%$ pentobarbital solution by intraperitoneal injection, and then sacrificed by bleeding through the aorta. The carotid arteries were rapidly removed and cleared. One sample of the carotid artery was fixed in $4 \%$ paraformaldehyde for immunohistochemical analysis, and another sample was frozen at $-80^{\circ} \mathrm{C}$ for quantitative reverse transcription polymerase chain reaction (qPCR) and western blotting.

ELISA analysis of serum malondialdehyde (MDA) and superoxide dismutase (SOD) levels. MDA and SOD levels were assessed before sacrificing the animals in all groups, using a commercially available MDA colorimetric assay kit and SOD colorimetric assay kit according to the manufacturer's protocol (ELISA kits; Nanjing Jiancheng Bioengineering Institute). The manufacturer's instructions were followed.

Immunohistochemical analysis of TRX protein. The carotid arteries were cleared, fixed in $4 \%$ formaldehyde, and processed in an automated Netherlands FEI Tecnai G2 Spirit $200 \mathrm{kV}$ machine (FEI Company, Eindhoven, The Netherlands) following paraffin embedding and preparation of $5-\mu \mathrm{m}$ sections.

The sections were oven-baked at $65^{\circ} \mathrm{C}$ for $2 \mathrm{~h}$, and washed three times with phosphate-buffered saline (PBS). The antigen was then repaired by quick cooling for 3 min after the highly compressed heating. Prior to staining, the sections were deparaffinized and incubated in $3 \%$ hydrogen peroxide for $10 \mathrm{~min}$ to quench any endogenous peroxidase. Nonspecific antibody binding sites were blocked with $10 \%$ normal goat serum in PBS. The section was then incubated overnight with the primary antibody: rabbit polyclonal to Thioredoxin/TRX (anti-TRX, 1:500; ab26320; Abcam, Cambridge, MA, USA) in blocking solution at $4^{\circ} \mathrm{C}$. The next day, the sections were washed three times with PBS, and incubated with a biotinylated goat anti-rabbit polyclonal antibody at room temperature for $60 \mathrm{~min}$. A horseradish peroxidase (HRP)-labeled streptavidin detection system was added for antigen staining at $37^{\circ} \mathrm{C}$ for $30 \mathrm{~min}$. Following five further washes with PBS and diaminobenzidine coloration, the sections were processed by hematoxylin staining for $2 \mathrm{~min}$, followed by bluing, dehydration, clearing and mounting. At this stage, morphologic changes in the vessel walls were visible, and images were recorded under a Nikon microscope (model E400; Nikon Inc., Melville, NY, USA).

qPCR analysis of TRX, TXNIP and VCAM-1 mRNA expression. Carotid arteries were frozen at $-80^{\circ} \mathrm{C}$ for $\mathrm{qPCR}$. The relative gene expression levels (the amount of target, normalized to endogenous control gene) were calculated using the comparative $\mathrm{Ct}$ method formula $2^{-\Delta \Delta \mathrm{CT}}$. The total RNA was extracted from the carotid artery using the SV Total RNA Isolation system (Promega Corp., Madison, WI, USA). For qPCR analyses, aliquots of total RNA were 
Table I. Fasting blood glucose and weight in each group.

\begin{tabular}{lrr}
\hline Groups & Fasting blood glucose (mmol/l) & Weight $(\mathrm{g})$ \\
\hline Normal control $(\mathrm{n}=6)$ & $4.900 \pm 0.433$ & $376.760 \pm 39.810$ \\
Diabetic control $(\mathrm{n}=6)$ & $20.480 \pm 2.011$ & $263.730 \pm 30.410$ \\
Nicorandil treated $(\mathrm{n}=5)$ & $20.370 \pm 1.917$ & $305.080 \pm 16.872$ \\
\hline
\end{tabular}
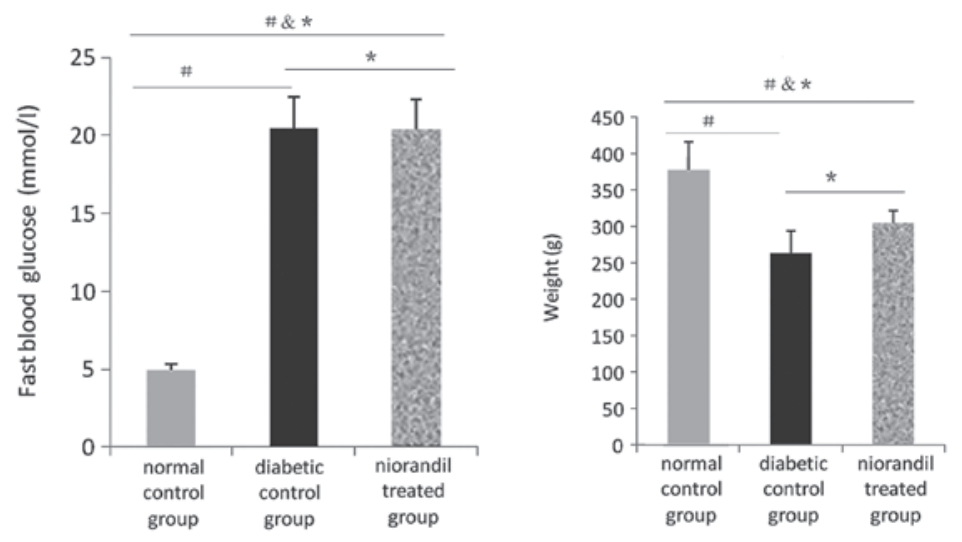

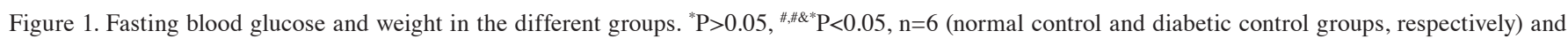
$\mathrm{n}=5$ (nicorandil-treated group).

reverse transcribed using a random hexamer primer. qPCR was performed using Power SYBR Green PCR Master Mix reagents (Applied Biosystems, Foster City, CA, USA). PCR cycling conditions were in accordance with the Power SYBR Green PCR Master Mix instructions: Initial denaturation at $95^{\circ} \mathrm{C}$ for $10 \mathrm{~min}$, followed by 40 cycles at $94^{\circ} \mathrm{C}$ for $10 \mathrm{sec}$, and $60^{\circ} \mathrm{C}$ for $1 \mathrm{~min}$. The primer pairs used were: $\beta$-actin, forward 5'-CACGATGGAGGGGCCGGACTCATC-3' and reverse 5'-TAAAGACCTCTATGCCAACACAGT-3' (241 bp); TRX, forward 5'-GCTGATCGAGAGCAAGGAAG-3' and reverse 5'-TCAAGGAACACCACATTGGA-3' (159 bp); TXNIP, forward 5'-ACCAGTGTCTGCCAAAAAGG-3' and reverse 5'-GCCATTGGCAAGGTAAGTGT-3' (202 bp); VCAM-1, forward 5'-ACAAAACGCTCGCTCAGATT-3' and reverse 5'-GTCCATGGTCAGAACGGACT-3' (152 bp). The cDNA standard sample was replaced with water in all sample controls.

Western blot analysis of TRX protein. TRX protein levels in carotid arteries were determined using western blot analysis. Carotid arteries, which had been frozen in liquid nitrogen immediately following isolation and stored at $-80^{\circ} \mathrm{C}$, were homogenized in $25 \mathrm{mM}$ Tris- $\mathrm{HCl}$ ( $\mathrm{pH} 7.4), 1 \mathrm{mM}$ dithiolthreitol, $1 \mathrm{mM}$ sodium orthovanadate, a protease inhibitor cocktail tablet, phosphatase inhibitor cocktail and $1 \%$ Triton X-100, using a homogenizer. The homogenates were centrifuged at $15,000 \mathrm{x} \mathrm{g}$ for $20 \mathrm{~min}$ at $4^{\circ} \mathrm{C}$. The supernatants were collected, and protein concentrations were determined using a bicinchoninic acid (BCA) Protein Assay kit (Thermo Fisher Scientific, Inc., Waltham, MA, USA). Equal amounts of protein extracts were separated on a $12 \%$ SDS-polyacrylamide gel and immobilized on polyvinylidene difluoride (PVDF) membranes (Millipore, Billerica, MA, USA). The membranes were blocked in PVDF blocking reagent (Toyobo, Osaka, Japan) and incubated with 1:400 anti-TRX antibodies (Abcam). Following washing, the membranes were incubated with anti-rabbit immunoglobulin $\mathrm{G}$ ( $\mathrm{IgG}$ ) polyclonal conjugated with HRP (1:10,000; Wuhan Boster Biological Technology Ltd., Wuhan, China). Immunoreactive signals were visualized using SuperSignal West Dura Extended Duration Substrate (Thermo Fisher Scientific, Inc.), and detected using a Kodak XRS system (Eastman Kodak, Fair Lawn, NJ, USA). Each protein signal was normalized to the $\beta$-actin expression in the same sample.

Statistical analysis. Data are presented as the mean \pm standard deviation. A comparison of the means was performed using Student's t-test for two groups, and a one-way analysis of variance (ANOVA) was applied to multiple groups with SPSS 17.0 (SPSS Inc., Chicago, IL, USA). $\mathrm{P}<0.05$ was considered to indicate a statistically significant difference.

\section{Results}

Effect of nicorandil on fasting blood glucose levels and weight. Fasting blood glucose levels in the diabetic group receiving nicorandil were $>14 \mathrm{mmol} / \mathrm{l}$. In the diabetic control group, the average glucose level was 20.48 \pm 2.011 versus $4.90 \pm 0.433 \mathrm{mmol} / 1$ in the normal control group $(\mathrm{P}<0.05)$. In the nicorandil treated group, the average glucose level was $20.37 \pm 1.917$ versus $20.48 \pm 2.011 \mathrm{mmol} / 1$ in the diabetic control group $(\mathrm{P}>0.05)$. There were also differences in weight between the diabetic control and normal control groups $(376.76 \pm 39.810$ versus $263.73 \pm 30.410 \mathrm{~g}, \mathrm{P}<0.05)$, and 

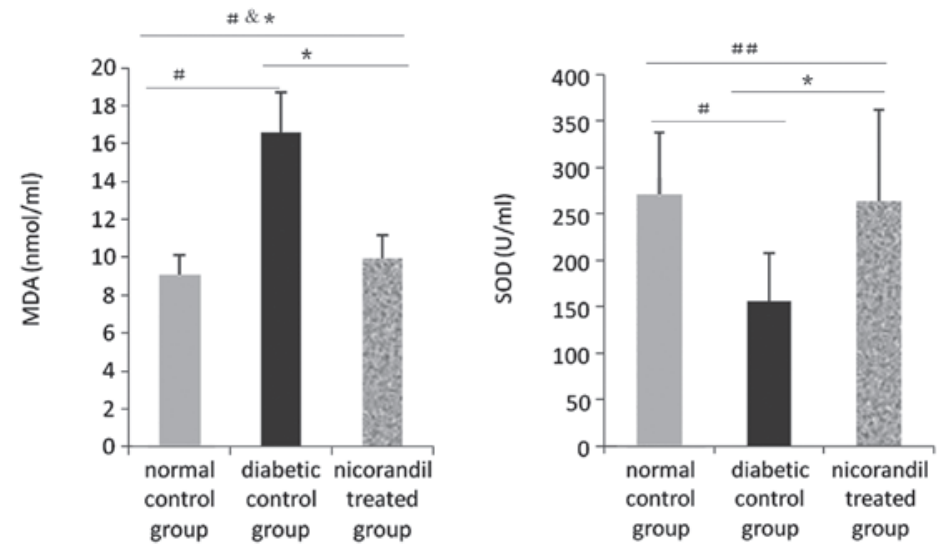

Figure 2. Levels of serum MDA and SOD in the different groups. ${ }^{\# \#} \mathrm{P}>0.05,{ }^{*}, \#, \# \notin *{ }^{*} \mathrm{P}<0.05, \mathrm{n}=6$ (normal control and diabetic control groups, respectively) and $\mathrm{n}=5$ (nicorandil-treated group). MDA, malondialdehyde; SOD, superoxide dismutase.
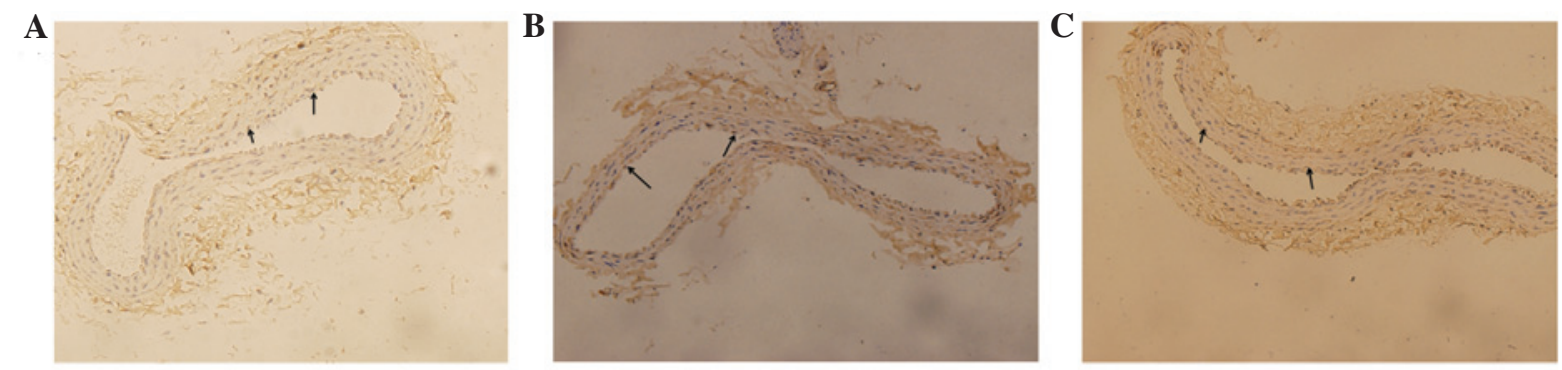

Figure 3. Immunohistochemistry of thioredoxin (TRX) protein in all groups (A, normal control group; B, diabetic control group; C, nicorandil-treated group). (A) Normal control group: Blue staining indicates cytoblasts, brown staining surrounding the cytoblast indicates positive expression of TRX protein. (B) Diabetic control group: Brown staining around the cytoblasts is reduced compared with the normal control and nicorandil-treated groups, indicating that the expression of TRX is decreased in diabetic rats. (C) Nicorandil-treated group: Brown staining around the cytoblast is reduced compared with the normal control group; however, it is increased compared with the diabetic control group, indicating that nicorandil may upregulate the expression of TRX. Magnification, x200. Image analysis was performed with Image-Pro Plus 6.0 software (Media Cybernetics, Inc., Rockville, USA).

between the nicorandil-treated and diabetic control groups $(305.08 \pm 16.872$ versus $263.73 \pm 30.410 \mathrm{~g}, \mathrm{P}>0.05)$. These results indicate that the diabetic animal model was successful and the level of oxidative stress is more serious in diabetic than in normal rats. Treatment with nicorandil may not worsen the hyperglycemic status in diabetic rats, but may improve the redox-active reaction in diabetic rats, as fasting blood glucose levels in the nicorandil group were lower than those in the diabetic control group, although this difference was not statistically significant ( $\mathrm{P}>0.05)$ (Table I and Fig. 1).

Effect of nicorandil on the serum levels of MDA and SOD. SOD basal levels were determined in animals from the normal control group $(270.200 \pm 66.856 \mathrm{U} / \mathrm{ml})$. Compared with the SOD baseline, the SOD level in the diabetic control group was decreased $(270.200 \pm 66.856$ versus $156.309 \pm 51.243 \mathrm{U} / \mathrm{ml}, \mathrm{P}<0.05)$. The diabetic status may have induced higher levels of oxidative stress. Following nicorandil administration, the SOD serum level was higher than in the diabetic control group (262.756 \pm 99.264 versus $156.309 \pm 51.243 \mathrm{U} / \mathrm{ml}, \mathrm{P}<0.05)$. Nicorandil may lower the level of oxidative stress. Simultaneously, MDA serum levels may also reflect this phenomenon. MDA in the normal control group was at the baseline level $(9.067 \pm 1.086 \mathrm{nmol} / \mathrm{ml})$, but was increased in the diabetic control group $(9.067 \pm 1.086$ versus

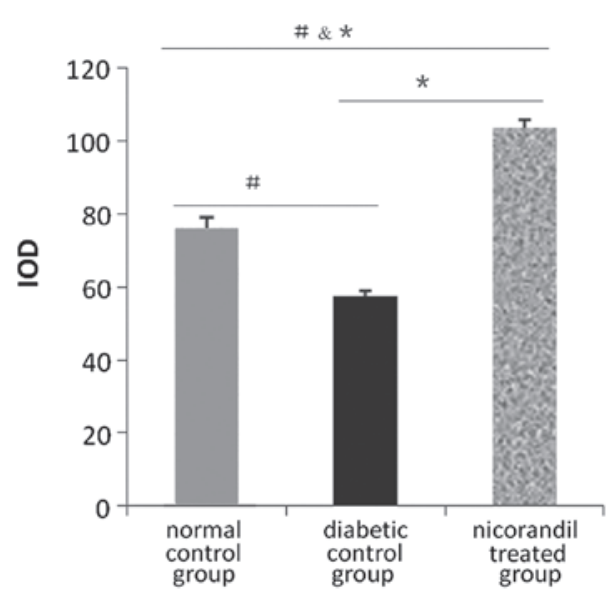

Figure 4. Analysis of TRX expression using IPP in the different groups. At least five images for each artery in the three groups were used for analysis. $*, \#, \# \&$ $\mathrm{P}<0.05, \mathrm{n}=6$ (normal control and diabetic control groups, respectively) and $n=5$ (nicorandil-treated control group). IOD, integrated optical density.

$16.620 \pm 2.101 \mathrm{nmol} / \mathrm{ml}, \mathrm{P}<0.05)$. Nicorandil may lower oxidative stress through its combined effects; compared with the diabetic control group, the serum MDA level was decreased following nicorandil administration $(9.96 \pm 1.228$ versus $16.620 \pm 2.101 \mathrm{nmol} / \mathrm{ml}, \mathrm{P}<0.05$ ) (Fig. 2). 

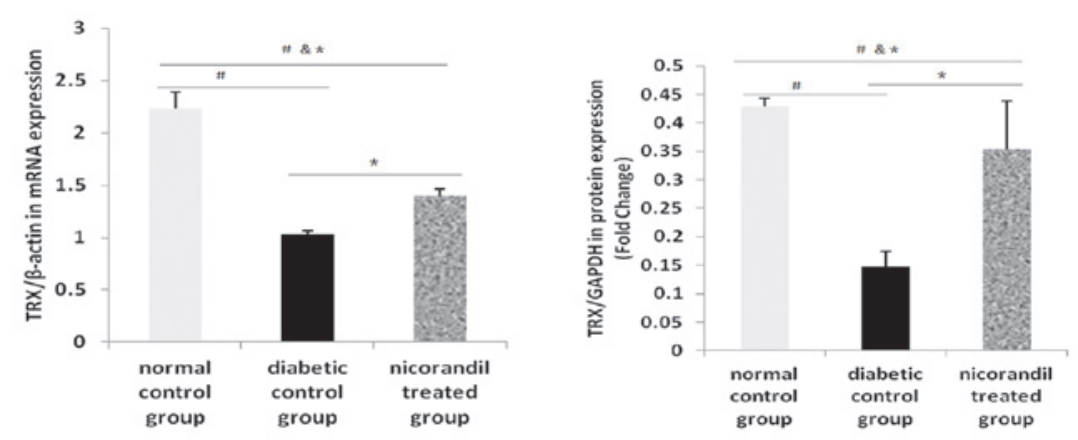

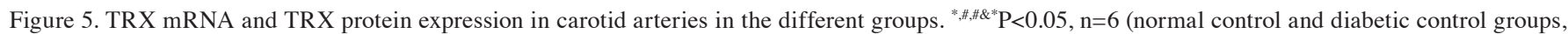
respectively) and $\mathrm{n}=5$ (nicorandil-treated group). TRX, thioredoxin.
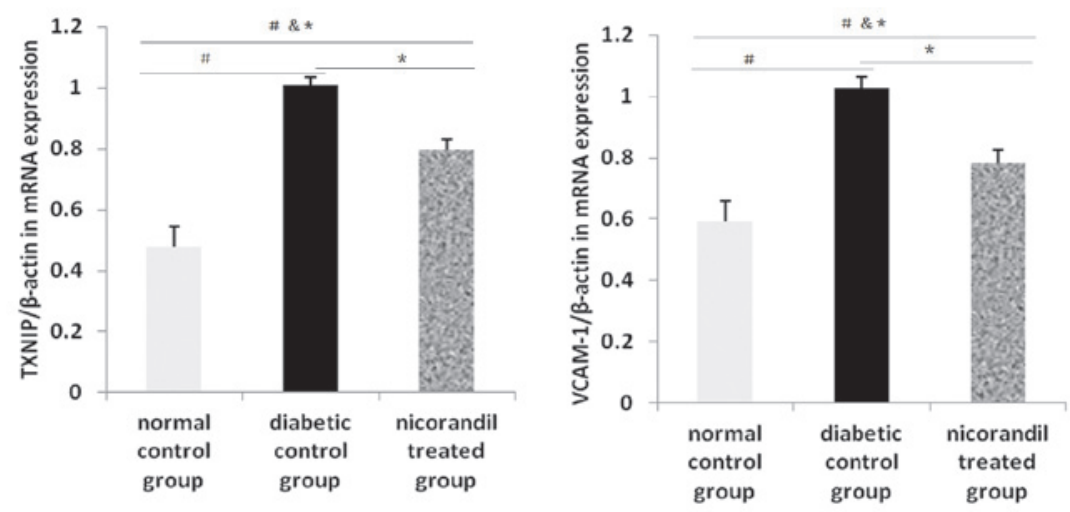

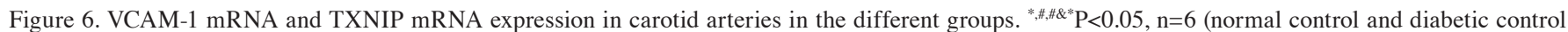
groups, respectively) and n=5 (nicorandil-treated group). VCAM-1, vascular cell adhesion molecule-1; TXNIP, thioredoxin interacting protein.

Immunohistochemical assessment of the effect of nicorandil on the protein expression of TRX

Immunohistochemistry images. Fig. 3A-C shows TRX expression levels in the carotid arteries. Positive expression of TRX protein is indicated by a blue staining of the endothelial cell nucleus and a brown stain of the area surrounding the cell nucleus. Endothelial cells only staining blue are negative for TRX expression.

qPCR and western blot analysis of the effect of nicorandil on the expression of TRX. Compared with the normal control group, TRX mRNA levels were decreased in the diabetic control group $(2.234 \pm 0.155$ versus $1.026 \pm 0.043)$. In the nicorandil treated group, TRX mRNA levels were higher than those in the diabetic control group $(1.395 \pm 0.066$ versus $1.026 \pm 0.043$ ), similar to the immunohistochemistry results. The expression levels of TRX protein in the diabetic control group were the lowest compared with the normal control group $(0.147 \pm 0.0263$ versus $0.464 \pm 0.0176)$ and the nicorandil-treated group $(0.147 \pm 0.0263$ versus $0.333 \pm 0.0807)$ (Fig. 5). This indicates that the diabetic status may worsen the levels of oxidative stress in vivo and inhibit the expression of producers of antioxidants (Fig. 6). The differences in TRX protein expression levels between the groups were statistically significant $(\mathrm{P}<0.05, \mathrm{n}=5-6)$.
Effect of nicorandil on the expression levels of TXNIP and VCAM-1 mRNA. The level of VCAM-1 mRNA expression was increased in the diabetic control group compared with that in the normal control group $(0.592 \pm 0.068$ versus $1.026 \pm 0.038$, $\mathrm{P}<0.05)$ and the nicorandil-treated group $(1.026 \pm 0.038$ versus $0.780 \pm 0.046, \mathrm{P}<0.05)$. Diabetes may increase the levels of factors inducing oxidation and promote endothelial cell dysfunction.

mRNA expression levels of TXNIP were also assessed in order to demonstrate the change in diabetic status and the influence of nicorandil. TXNIP mRNA levels in the diabetic control group were significantly higher than in the other groups. Compared with the normal control group $(1.007 \pm 0.026$ versus $0.478 \pm 0.066, \mathrm{P}<0.05)$ and the nicorandil-treated group $(1.007 \pm 0.026$ versus $0.798 \pm 0.033, \mathrm{P}<0.05)$, the production of factors increasing oxidation was increased in the diabetic control group (Fig. 6).

\section{Discussion}

The results of this study indicate that nicorandil attenuates the formation of ROS, and that the decrease in TXNIP levels appears to be involved in the expression of TRX protein, consequently resulting in the suppression of VCAM-1 secretion in STZ-induced diabetic vascular endothelial cells of 
rats. Endothelial cells release a variety of inflammatory mediators and adhesion molecules in atherosclerosis and diabetes, including VCAM-1, intercellular adhesion molecule (ICAM-1), as well as P- and E-selectins (16). These are membrane proteins necessary for anchoring leukocytes to the vessel wall and act as biomarkers of endothelial dysfunction under inflammatory conditions. In particular, VCAM-1 has been revealed to be an important marker in diabetes. Increased levels of VCAM-1 have been detected in the serum and vitreous of patients with diabetes. Moreover, serum levels of VCAM-1 and E-selectin are increased in patients with microand macro-vascular complications (17-19). The present study demonstrates that nicorandil treatment attenuates VCAM-1 expression in an STZ-induced model of diabetes in rats. The mechanism underlying this regulatory effect of nicorandil in endothelial cells warrants further investigation. Current research indicates that nicorandil reduces strain-induced ROS generation (20), scavenges radical species (15) and normalizes NADPH oxidase and NOS in STZ-induced diabetic rats (14). Nicorandil is a potent antioxidant. The present study demonstrates that administered nicorandil suppresses VCAM-1 gene expression, accompanied by a decrease in TXNIP levels and ROS formation. Consequently, one possible explanation for the inhibitory effect of nicorandil may be its ability to attenuate redox-active agents. Alternatively, nicorandil may inhibit the gene expression of TXNIP by increasing TRX protein expression.

As previously described, TRX systems may have an important role in redox reactions. TRX reduces the oxidized form of TRX peroxidase, and the reduced TRX peroxidase then scavenges ROS. TRX exerts the majority of its antioxidant properties in this manner $(21,22)$. Nicorandil-induced increased levels of TRX expression may further impede the production of redox-active agents, including the gene expression of TXNIP and VCAM-1.

Although various studies on the effects of nicorandil and several types of agents in redox reactions are available, the effect of nicorandil on TRX expression remains to be investigated. The present study indicates that nicorandil may attenuate VCAM-1 gene expression in an STZ model of diabetes in rats via increasing the TRX expression.

\section{References}

1. Cai L and Kang YJ: Oxidative stress and diabetic cardiomyopathy: a brief review. Cardiovasc Toxicol 1: 181-193, 2001.

2. Schaffer SW, Jong CJ and Mozaffari M: Role of oxidative stress in diabetes-mediated vascular dysfunction: Unifying hypothesis of diabetes revisited. Vascul Pharmacol 57: 139-149, 2012.

3. Schulze PC, Yoshioka J, Takahashi T, He Z, King GL and Lee RT: Hyperglycemia promotes oxidative stress through inhibition of thioredoxin function by thioredoxin-interacting protein. J Biol Chem 279: 30369-30374, 2004.
4. Mahmood DF, Abderrazak A, El Hadri K, Simmet T and Rouis M: The thioredoxin system as a therapeutic target in human health and disease. Antioxid Redox Signal 19: 1266-1303, 2013.

5. Hwang J, Suh HW, Jeon YH, et al: The structural basis for the negative regulation of thioredoxin by thioredoxin-interacting protein. Nat Commun 5: 2958, 2014.

6. Dunn LL, Buckle AM, Cooke JP and Ng MK: The emerging role of the thioredoxin system in angiogenesis. Arterioscler Thromb Vasc Biol 30: 2089-2098, 2010.

7. Yamawaki H, Pan S, Lee RT and Berk BC: Fluid shear stress inhibits vascular inflammation by decreasing thioredoxin-interacting protein in endothelial cells. J Clin Invest 115: 733-738, 2005.

8. IONA study group: Effect of nicorandil on coronary events in patients with stable angina: the Impact Of Nicorandil in Angina (IONA) randomised trial. Lancet 359: 1269-1275, 2002.

9. Horinaka S, Yabe A, Yagi H, et al: Effects of nicorandil on cardiovascular events in patients with coronary artery disease in the Japanese Coronary Artery Disease (JCAD) study. Circ J 74: 503-509, 2010.

10. Walker A, McMurray J, Stewart S, et al: Economic evaluation of the impact of nicorandil in angina (IONA) trial. Heart 92: 619-624, 2006

11. Taira N: Nicorandil as a hybrid between nitrates and potassium channel activators. Am J Cardiol 63: 18J-24J, 1989.

12. Reimann F, Ashcroft FM and Gribble FM: Structural basis for the interference between nicorandil and sulfonylurea action. Diabetes 50: 2253-2259, 2001.

13. Kasono K, Yasu T, Kakehashi A, et al: Nicorandil improves diabetes and rat islet beta-cell damage induced by streptozotocin in vivo and in vitro. Eur J Endocrinol 151: 277-285, 2004.

14. Serizawa K, Yogo K, Aizawa K, Tashiro Y and Ishizuka N: Nicorandil prevents endothelial dysfunction due to antioxidative effects via normalisation of NADPH oxidase and nitric oxide synthase in streptozotocin diabetic rats. Cardiovasc Diabetol 10: 105, 2011.

15. Mano T, Shinohara R, Nagasaka A, et al: Scavenging effect of nicorandil on free radicals and lipid peroxide in streptozotocin-induced diabetic rats. Metabolism 49: 427-431, 2000.

16. Khan ZA and Chakrabarti S: Cellular signaling and potential new treatment targets in diabetic retinopathy. Exp Diabetes Res 2007: 31867, 2007.

17. Adamiec-Mroczek J and Oficjalska-Młyńczak J: Assessment of selected adhesion molecule and proinflammatory cytokine levels in the vitreous body of patients with type 2 diabetes - role of the inflammatory-immune process in the pathogenesis of proliferative diabetic retinopathy. Graefes Arch Clin Exp Ophthalmol 246: 1665-1670, 2008

18. Gustavsson C, Agardh CD, Zetterqvist AV, Nilsson J, Agardh E and Gomez MF: Vascular cellular adhesion molecule-1 (VCAM-1) expression in mice retinal vessels is affected by both hyperglycemia and hyperlipidemia. PLoS One 5: e12699, 2010.

19. Limb GA, Hickman-Casey J, Hollifield RD and Chignell AH: Vascular adhesion molecules in vitreous from eyes with proliferative diabetic retinopathy. Invest Ophthalmol Vis Sci 40: 2453-2457, 1999.

20. Chao HH, Hong HJ, Sung LC, Chen JJ, Cheng TH and Liu JC: Nicorandil attenuates cyclic strain-induced endothelin-1 expression via the induction of activating transcription factor 3 in human umbilical vein endothelial cells. Eur J Pharmacol 667: 292-297, 2011.

21. Chae HZ, Chung SJ and Rhee SG: Thioredoxin-dependent peroxide reductase from yeast. J Biol Chem 269: 27670-27678, 1994.

22. World CJ, Yamawaki H and Berk BC: Thioredoxin in the cardiovascular system. J Mol Med (Berl) 84: 997-1003, 2006. 